\title{
2/3D imaging based on photonics-enabled multi-band MIMO radar system
}

\author{
Antonella Bogoni ${ }^{1,2}$, Salvatore Maresca ${ }^{1}$, David Ricardo Sanchez Jacome ${ }^{1,4}$, Filippo Scotti ${ }^{2}$, Giovanni Serafino ${ }^{1}$, \\ Antonio Malacarne ${ }^{1}$, Leonardo Lembo ${ }^{1,3}$, Carsten Rockstuhl ${ }^{4}$, Paolo Ghelfi ${ }^{2}$ \\ 1 Scuola Superiore Sant'Anna, Via Moruzzi 1, 56124 Pisa, Italy, Email: antonella.bogoni@cnit.it \\ 2 CNIT, Via Moruzzi 1, 56124 Pisa, Italy \\ 3 Italian Navy, Naval Research Center CSSN, Vallauri Institute, Viale Italia 72, 57100 Livorno, Italy \\ 4 Karlsruhe Institute of Technology, Germany
}

\begin{abstract}
Photonics-enabled coherent MIMO radars have been numerically investigated to evaluate the benefits of coherence and multi-band operation in 2/3D imaging.
\end{abstract}

\section{Keywords-mircowave photonics, 5G, radar}

Multiband and distributed radars are essential for accurate and reliable $2 / 3 \mathrm{D}$ imaging of targets. (i) Multiband radars, working at different radio frequencies (RF) improve the system detection capability and reliability, making the system more robust to the environmental conditions and target scattering features. Coherent data fusion among multiband detections allows to avoid the loss of data and to optimize the system precision. Unfortunately, conventional RF electronics are intrinsically narrow band and lack in flexibility. Therefore, multiband radars can be obtained only by using several independent and non-coherent single-band apparatuses. In these cases, coherence among data is digitally reconstructed in the radar processing through heavy synchronization algorithms with high computational complexity. (ii) Moreover, distributed multiple input-multiple output (MIMO) radars are systems employing multiple transmit waveforms that have the ability to jointly process signals received by multiple antennas. They allow the scene observation from different viewpoints, improving the capability to detect targets characterized by high angular radar cross section variability (e.g. complex or stealth targets). MIMO radars also allow to increase the cross-range (i.e. angular) resolution. In fact, in a monostatic radar the range resolution is determined by the bandwidth of the transmitted signal, whereas the cross-range resolution depends on the antenna beam aperture and the target distance. On the other hand, MIMO radars exploit spatially distributed information to achieve an excellent cross-range resolution independent of antenna features. Finally, the coherence of the data collected from different positions can increase the detection system performance. Unfortunately, the development of MIMO radars with widely separated antennas is nowadays accompanied by two main issues: they need high capacity data links to send raw data from the multiple antennas to the central processor; and they require extremely high phase coherence among all the radar signals, i.e. among all the components of the distributed system. Finding an RF solution to these problems is challenging. Recently, the use of photonics has been demonstrated both for radar signal generation and elaboration, and radar signal distribution providing distributed coherent multiband operation [1]-[3]. Here, the benefits of coherent distributed radar systems are numerically investigated.
As an example, Fig. 1 reports the coherent MIMO system performance in the presence of a single target, in the case of Tx and $\mathrm{Rx}$ antennas placed on a 1D 3m-long baseline thus enabling 2D imaging. In all the investigated cases the antenna positions have been optimized. The following configurations have been analyzed: a single-band $2 \mathrm{Tx} / 4 \mathrm{Rx}(2 \mathrm{x} 4)(\mathrm{a}, \mathrm{b})$ a single band $4 \times 8(c)$ and a dual-band $4 \times 8$ (d) configurations. The used RF carriers are $9 \mathrm{GHz}$ and $8 \& 9 \mathrm{GHz}$ in the case of single and dual-band operation respectively. The frequency modulated continuous wave signals have bandwidth $=400 \mathrm{MHz}$.
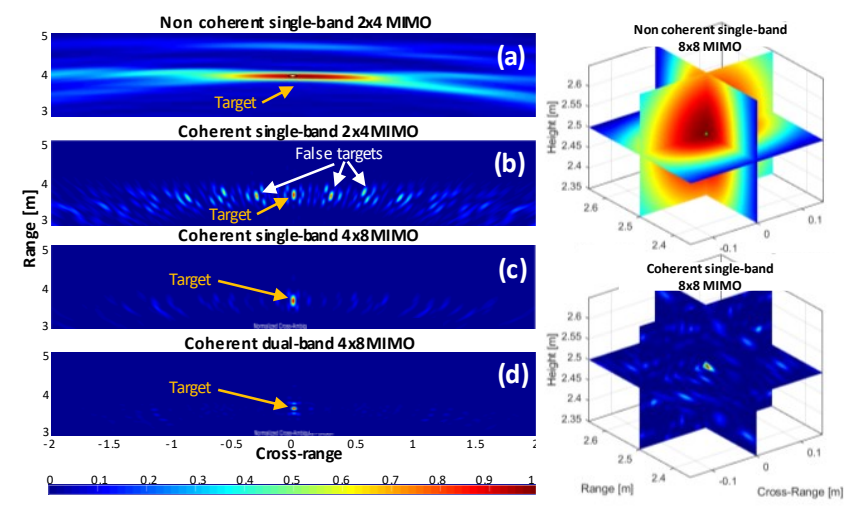

Fig. 1: Left: Range/cross-range map using a non-coherent single-band $2 \times 4$ (a), coherent single-band $2 \times 4$ (b), single-band $4 \times 8$ (c) and dual-band $4 \times 8$ (d) MIMO configuration. Right: Range/cross- range/height map using a non coherent (top) and coherent (bottom) single-band 8x8 MIMO configuration.

The benefits of coherence in terms of cross-range resolution is evident comparing Fig. 1(a) and (b)-(d) From a processing point of view, the coherent MIMO elaboration corresponds to a digital beamforming using a sparse antenna array. The sparse configuration of the antenna elements (i.e. the distributed antennas) results in the presence of side lobes that seriously affects the system performance leading to false target detection and localization errors (Fig. 1 b). However, these side lobes can be reduced by increasing the number of antennas (Fig. $1 \mathrm{c}$ ). Multiband operation can even further reduce the side lobes without changing sensor number (Fig. 1 d). 3D imaging has been also simulated in different configurations of practical interest. Fig. 1 (right) shows the case of a $8 \times 8$ configuration with a 2D 5m-long baseline to verify the benefits of coherence in both cross-range and height resolution.

[1] P. Ghelfi, et al., J. Lightwave Technol., vol. 34, n. 2, 2016

[2] P.Ghelfi et al, Nature Vol 507 March 2014.

[3] F.Laghezza et all, IET Radar Sonar \& Navig. Journal, Vol 9, n. 8, 2015. 